PTH-96 COLORECTAL CANCER DIAGNOSED AT SIGMOIDOSCOPY, IS COLONOSCOPY NECESSARY TO ASSESS FOR SYNCHRONOUS CANCER?

Vikram Mohanan, Ajay Verma*. Kettering General Hospital Nhs Foundation Trust, Kettering, $U K$

\subsection{6/gutjnl-2021-BSG.299}

Introduction The Association of Coloproctology of Great Britain \& Ireland (ACGBI) 2017 colorectal cancer (CRC) guidelines reaffirmed the longstanding practice of assessing for synchronous cancer in patients diagnosed with CRC at sigmoidoscopy (f-sig). Ideally by colonoscopy in addition to CT staging of chest, abdomen \& pelvis (CT CAP), or alternatively CT colonography (CTC) and CT thorax if complete colonoscopy not possible.

In the literature, approximately $3.5 \%$ of patients had synchronous CRC. Scheduling colonoscopy may delay treatment and be onerous for patients. Access to prompt colonoscopy can be challenging due to capacity issues, especially in the COVID-19 pandemic era.

Methods Data were retrospectively analysed from electronic endoscopy, radiology and pathology records from patients diagnosed with CRC at f-sig and colonoscopy over 11 years (2010-2020 inclusive).

Results Analysis 1: 680 patients who had CRC diagnosed at fsig: 230 underwent pre-treatment colonoscopy (33.8\%).

Interval between f-sig and colonoscopy; mean 17.5 days/ median 15.0 days.

Two synchronous cancers identified at colonoscopy; 0.9\%

1. 57 years old man with primary rectal cancer and synchronous transverse colon cancer - both lesions reported on staging imaging scans.

- 69 years old woman with a primary rectal cancer and synchronous sigmoid colon cancer (not seen at f-sig due to poor preparation) - both lesions reported on staging imaging scans.

Analysis 2: 796 patients who had CRC diagnosed at colonoscopy:

$48 / 796$ have a significant 2 nd finding (6.0\%)

- 24 had synchronous CRC (3.0\%)/24 had a significant polyp $>20 \mathrm{~mm}(3.0 \%)$

In these 48 cases, if F-sig was performed instead of colon, what would have been the outcome?

- Only in one case would a significant lesion be missed. 72 years old man with a primary rectal cancer and a $30 \mathrm{~mm}$ ascending colon polyp (not seen on staging CT scan).

- In the other 47 cases; staging CT scans pick up lesions or metastases, or lesions are all left sided and would be seen at F-sig, or lesions are all right sided and would not be seen at f-sig, or missed lesion was a benign polyp.

Conclusions This is a large analysis of 1476 patients diagnosed with CRC. Of the 796 diagnosed at colonoscopy, 6.0\% had a synchronous lesion (48 patients), 3.0\% had a synchronous CRC, only 1 patient would have had a missed lesion if they'd had a f-sig alone. Of the 680 patients diagnosed with CRC at f-sig, 230 had a colonoscopy (33.5\%), the rest were precluded due to advanced disease/obstruction or weren't fit due to advanced age/co-morbidity. Colonoscopy was undertaken at a median of 15.0 days. The yield of identifying a synchronous cancer at colonoscopy in this cohort is $<1 \%$, in both cases these lesions were reported on staging imaging scans.

British Society of Gastroenterology and ACPGBI guidelines from 2019 suggest that in patients who are fit/suitable they

\begin{tabular}{|c|c|c|c|c|c|}
\hline $\begin{array}{l}\text { Colonoscopy after } \\
\text { f-sig }\end{array}$ & $\begin{array}{l}\text { Number } \\
\text { (n) }\end{array}$ & $\begin{array}{l}\text { Female } \\
\text { (n) }\end{array}$ & Male (n) & $\begin{array}{l}\text { Mean age } \\
\text { (years) }\end{array}$ & $\begin{array}{l}\text { Median age } \\
\text { (years) }\end{array}$ \\
\hline No & 450 & 193 & $\begin{array}{l}257 \\
(57.1 \%)\end{array}$ & 73.2 & 75.5 \\
\hline Yes & 230 & 77 & $\begin{array}{l}153 \\
(66.5 \%)\end{array}$ & 66.8 & 67.8 \\
\hline Colonoscopy only & 796 & 320 & $\begin{array}{l}476 \\
(59.8 \%)\end{array}$ & 69.1 & 69.1 \\
\hline
\end{tabular}

should undergo a surveillance colonoscopy at 12 months post CRC diagnosis. Given the capacity issues affecting colonoscopy services in the pandemic era, a proposed pathway for patients diagnosed with CRC at sigmoidoscopy; if staging imaging scans shows resectable CRC without synchronous lesion, is to consider undergoing surgery and to utilise 12-month colonoscopy to clear any adenomas. Alternatively CTC and CT thorax could be utilised though capacity issues may limit this approach. This data supports the consideration of alternative approaches as the likelihood of a synchronous cancer not seen at sigmoidoscopy and staging imaging scan appears to be very low.

\section{PTH-97 FIT IN THE POST-COVID-19 COLORECTAL PATHWAY - ARE WE DOING IT RIGHT?}

${ }^{1}$ Hing Chi Kristie Leung*, 'Preena Patel, ${ }^{2}$ Edward Seward. ${ }^{1}$ UCL Medical School, Faculty of Medical Sciences, University College London, London, UK; ${ }^{2}$ Endoscopy Department, University College London Hospital, London, UK

\subsection{6/gutjnl-2021-BSG.300}

Introduction During COVID-19 pandemic, national guidance updated NG12 colorectal cancer (CRC) referral criteria to incorporate Faecal Immunochemical Testing (FIT), previously reserved only for patients on the low risk DG30 pathway. Positive FIT results $(\geq 10 \mu \mathrm{g} / \mathrm{g}$ of faeces) in patients with colorectal symptoms would trigger a two week wait $(2 \mathrm{ww})$ referral for urgent investigations. Otherwise, patients with 'negative FIT' were expected to be safety-netted but not investigated. Patients with high-risk symptoms (i.e. NG12 compliant) could be referred in the absence of FIT result. We wished to explore how this change has affected referral patterns and whether the new guidance was adhered to.

Methods We extracted 2ww colorectal referrals to University College London Hospital from August to October 2020. Demographics, reason for referral, symptoms, investigations and outcome were recorded. These values were compared between patients who had FIT (FIT group) to those who did not (no FIT group). Two-tailed t-test and Chi-squared test were used to assess for significant difference between the two groups.

Results 522 referrals were received in the period examined, 92 were excluded as they were either repeat referrals or contained insufficient clinical information. Of the remaining 420, 315 underwent FIT, although $37 \%$ of results were negative. Of those with a positive FIT, 73\% were NG12 compliant and $27 \%$ had neither NG12 nor DG30 symptoms. In the FIT negative group, 77\% were NG12 compliant. 105 patients were referred without FIT, of these 90\% were NG12 compliant. There was no significant difference in mean age (FIT $62 \mathrm{v}$ no 
FIT 62 years, $\mathrm{p}=0.10)$ and gender $(\mathrm{p}=0.41)$. Although the differences did not reach significance, advanced pathology in the FIT group was much higher, both in polyps $\geq 10 \mathrm{~mm}$ diameter (FIT $86 \%$ v no FIT $14 \%, \mathrm{p}=0.30$ ) and CRC incidence (FIT 4.5\% v no FIT 1.0\%, p = 0.19).

Conclusions Despite the challenges of managing clinical services during a pandemic, our data demonstrates an increased use of FIT in the colorectal pathway in line with updated guidance. This is particularly encouraging as there was insufficient time for an adequate communication strategy. However, a substantial proportion of patients were referred based on FIT results with neither NG12 nor DG30 compliant symptoms; perhaps showing that FIT can potentially increase referrals if used incorrectly. Some patients were referred with a negative FIT and a preponderance of NG12 symptoms, possibly indicating that clinical concern over traditional cancer symptoms may prompt referral despite an objectively very low cancer risk.

\section{PTH-98 FAECAL IMMUNOCHEMICAL TEST, FAST SCORE OR NG12 CRITERIA FOR DETECTION OF CANCER}

${ }^{1}$ Rigers Cama*, ${ }^{1}$ Neel Kapoor, 'Lefkothea Zacharopoulou, 'Leila Mebarek, 'Haroon Bhatti, ${ }^{2}$ Philip Sawyer, ${ }^{3}$ Bharat Patel, ${ }^{1} J o n a t h a n ~ L a n d y . ~{ }^{1}$ Gastroenterology Dept, West Hertfordshire Hospitals NHS Trust, Watford, WD18 OHB; ${ }^{2}$ Parkbury House Surgery, Herts Valleys CCG, St Albans, AL1 3HD; ${ }^{3}$ Chemical Pathology Dept, West Hertfordshire Hospitals NHS Trust, Watford, WD18 OHB

\subsection{6/gutjnl-2021-BSG.301}

Introduction Studies have evaluated FIT in patients meeting NG12 criteria suggesting greater accuracy for colorectal cancer (CRC) detection. The FAST score (faecal-Hb, age and sex test score) was proposed to improve the utility of $\mathrm{fHb}$ in the diagnosis of CRC. In 2019, Herts Valleys CCG instituted the use of FIT for patients in primary care presenting with symptoms meeting DG30 and some lower risk NG12 criteria (PPV $<3 \%$ ), excluding those with higher risk symptoms of iron deficiency anaemia (IDA), mass or rectal bleeding. We aimed to evaluate the utility of FIT with NG12 referral criteria and FAST score for the detection of CRC in our population.

Methods The medical records of all patients undertaking a FIT sample with a minimum of 6 months follow up between June 2019 and July 2020 were reviewed and cross referenced with the trust cancer database. Other outcomes recorded included inflammatory bowel disease and high-risk adenomas (defined as polyps of $\geq 1 \mathrm{~cm}, \geq 5$ polyps or high-grade dysplasia). FIT analysis was performed using a single OC-Sensor analyser (Eiken Chemical Co., Tokyo, Japan). FAST scores (> 2.12 versus $<2.12$ ) were calculated as previously described by Digby et al. (2019). Sensitivity, specificity, predictive values and numbers needed to investigate, were calculated using MedCalc ${ }^{\circledR}$ statistical software.

Results 3460 patients returned a FIT sample. The median age of population was 66 (IQR 56-76), with 57\% being female. 1046 patients underwent any investigation with 701 patients having full colonic evaluation. 22\% had FIT $>10 \mu \mathrm{g} / \mathrm{g}, 75 \%$ had FAST score $>2.12$ and 59\% met NG12 criteria. Sensitivity for CRC, in FIT, FAST group (> 2.12) and NG12 groups was 94\% (95\% CI 84-99\%), 100\% (95\% CI 93-100\%) and $82 \%$ (95\% CI 67-91\%) respectively. Specificity for CRC was $83 \%$ (95\% CI 82-84\%), 25\% (95\% CI 24-27\%) and $42 \%$ (95\% CI 40-43\%) respectively. The number needed to investigate to detect a patient with cancer was 16,52 and 50 for
FIT $>10 \mu \mathrm{g} / \mathrm{g}, \quad$ FAST score $>2.12$ and NG12 criteria respectively.

Conclusions FAST score $>2.12$ has the best sensitivity for detection of cancer. However, the specificity is low and significantly more patients would require investigation. FIT $>10$ $\mu \mathrm{g} / \mathrm{g}$ performed better than NG12 criteria.

\section{PTH-99 FAECAL IMMUNOCHEMICAL TESTSFOR YOUNGER PATIENTS PRESENTING WITH BOWEL SYMPTOMS}

${ }^{1}$ Lefkothea Zacharopoulou*, ${ }^{1}$ Rigers Cama, ${ }^{1}$ Neel Kapoor, 'Leila Mebarek, ${ }^{1}$ Haroon Bhatti, ${ }^{2}$ Philip Sawyer, ${ }^{3}$ Bharat Patel, 'Jonathan Landy. ${ }^{1}$ Gastroenterology Dept, West Hertfordshire Hospitals NHS Trust, Watford, WD18 OHB; ${ }^{2}$ Parkbury House Surgery, Herts Valleys CCG, St Albans, AL1 3HD; ${ }^{3}$ Chemical Pathology Dept, West Hertfordshire Hospitals NHS Trust, Watford, WD18 OHB

\subsection{6/gutjnl-2021-BSG.302}

Introduction Quantitative faecal immunochemical tests (FIT) are recommended by NICE (DG30) guidelines for use in patients with suspected colorectal cancer in primary care. However, the utility of FIT in patients under the age of 50 versus the use of faecal calprotectin is unclear. In 2019, Herts Valleys CCG instituted the use of FIT for patients over the age of 40 years, presenting with symptoms meeting DG30 and some lower risk NG12 criteria, excluding those with higher risk symptoms of iron deficiency anaemia (IDA), mass or rectal bleeding. We aim to evaluate the accuracy of FIT for significant bowel disease (SBD) in patients under 50 years with those 50-59 and over 60 years in our population.

Methods The medical records of all patients undertaking a FIT sample with a minimum of 6 months follow up between June 2019 and July 2020 were reviewed. The outcome of SBD (a composite of either colorectal cancer, inflammatory bowel disease or high-risk adenomas (defined as polyps of $\geq 1 \mathrm{~cm}, \geq 5$ polyps or high-grade dysplasia) was recorded. FIT analysis was performed using a single OC-Sensor io analyser (Eiken Chemical Co., Tokyo, Japan). The sensitivity, specificity, predictive values and accuracy of FIT for SBD were assessed for each age group. Fisher's exact test was used to assess the differences in sensitivity and specificity of FIT between patients $<50$ and older age groups. MedCalc ${ }^{\circledR}$ statistical software was used for all calculations.

Results 3460 patients with bowel symptoms undertook a FIT sample. 132 patients had SBD. 13\% of patients were $<50$ with a FIT result $\geq 10 \mu \mathrm{g} / \mathrm{g}$ in $12 \%$. $22 \%$ were $50-59$ with a FIT result $\geq 10 \mu \mathrm{g} / \mathrm{g}$ in $16 \%$. $65 \%$ were $\geq 60$ with a FIT result $\geq 10 \mu \mathrm{g} / \mathrm{g}$ in $26 \%$. The sensitivity, specificity, PPV, NPV and accuracy of a FIT result $\geq 10 \mu \mathrm{g} / \mathrm{g}$ for patients 50-59 were $85 \%$ (CI 66 to 95.8\%), 87\% (CI 84 to 89\%), 19\% (CI $15.8 \%$ to $23 \%$ ), $99.4 \%$ (CI $98.5 \%$ to $99.8 \%$ ) and $87 \%$ (CI $84 \%$ to $89 \%$ ) and for patients $\geq 60$ years were $86 \%$ (CI 77 to $92 \%$ ), $77 \%$ (CI 75 to $78.5 \%$ ), $14 \%$ (CI 13 to $16 \%$ ), $99.2 \%$ (CI $98.7 \%$ to $99.5 \%$ ) and $77 \%$ (CI $75 \%$ to $79 \%$ ). The sensitivity, specificity, PPV, NPV and accuracy of a FIT result $\geq 10 \mu \mathrm{g} / \mathrm{g}$ for patients $<50$ years were $87.5 \%$ (CI 47 to 99.7\%) $\mathrm{p}=1.0,89 \%$ (CI 85.7 to $91.9 \%$ ) $\mathrm{p}=0.35$ and $<0.01$, $13 \%$ (CI 9 to $17.8 \%$ ), $99.7 \%$ (CI 98.4 to $99.96 \%$ ) and $89 \%$ (CI 86 to $92 \%$ ) respectively.

Conclusion FIT performed well for the detection of SBD in all age groups with equivalent sensitivity between age groups and improved specificity in younger age groups compared with patients $\geq 60$ years. 